\title{
First-Principles Collision Cross Section Measurements of Large Proteins and Protein Complexes
}

Jacob W. McCabe, Christopher S. Mallis, Klaudia I. Kocurek, Michael L. Poltash, Mehdi Shirzadeh, Michael J. Hebert, Liqi Fan, Thomas E. Walker, Xueyun Zheng, ${ }^{\dagger}$ Ting Jiang, Shiyu Dong, Cheng-Wei Lin, Arthur Laganowsky, David H. Russell*

Department of Chemistry, Texas A\&M University, College Station, Texas 77843

${ }^{\dagger}$ Present address: Pacific Northwest National Laboratory, Richland, WA 99352

\# Present address: Beijing Institute of Technology, Beijing, China

*Email: Russell@chem.tamu.edu

\section{Supporting Information:}

\section{Tables:}

Table S1. Protein list with source $\quad$ S3

Table S2. CCS and $\mathrm{K}_{\mathrm{o}}$ values for PF-DT in helium collision gas $\quad$ S4

Table S3. GroEL MS data for various instrument configurations and tune conditions $\quad$ S10

\section{Figures:}

Figure S1. Example of SIMION determination of $\alpha$ for a single pressure $\quad$ S2

Figure S2. High Resolution MS data of BSA with and without chelator DTPA $\quad$ S6

Figure S3. High Resolution MS data of BSA under various AmA concentrations $\quad$ S7

Figure S4. High Resolution MS data of BSA with varying CE in the HCD cell. $\quad$ S8

Figure S5. High Resolution MS data of PK showing impurities in sample $\quad$ S9

$\begin{array}{ll}\text { References } & \text { S10 }\end{array}$ 


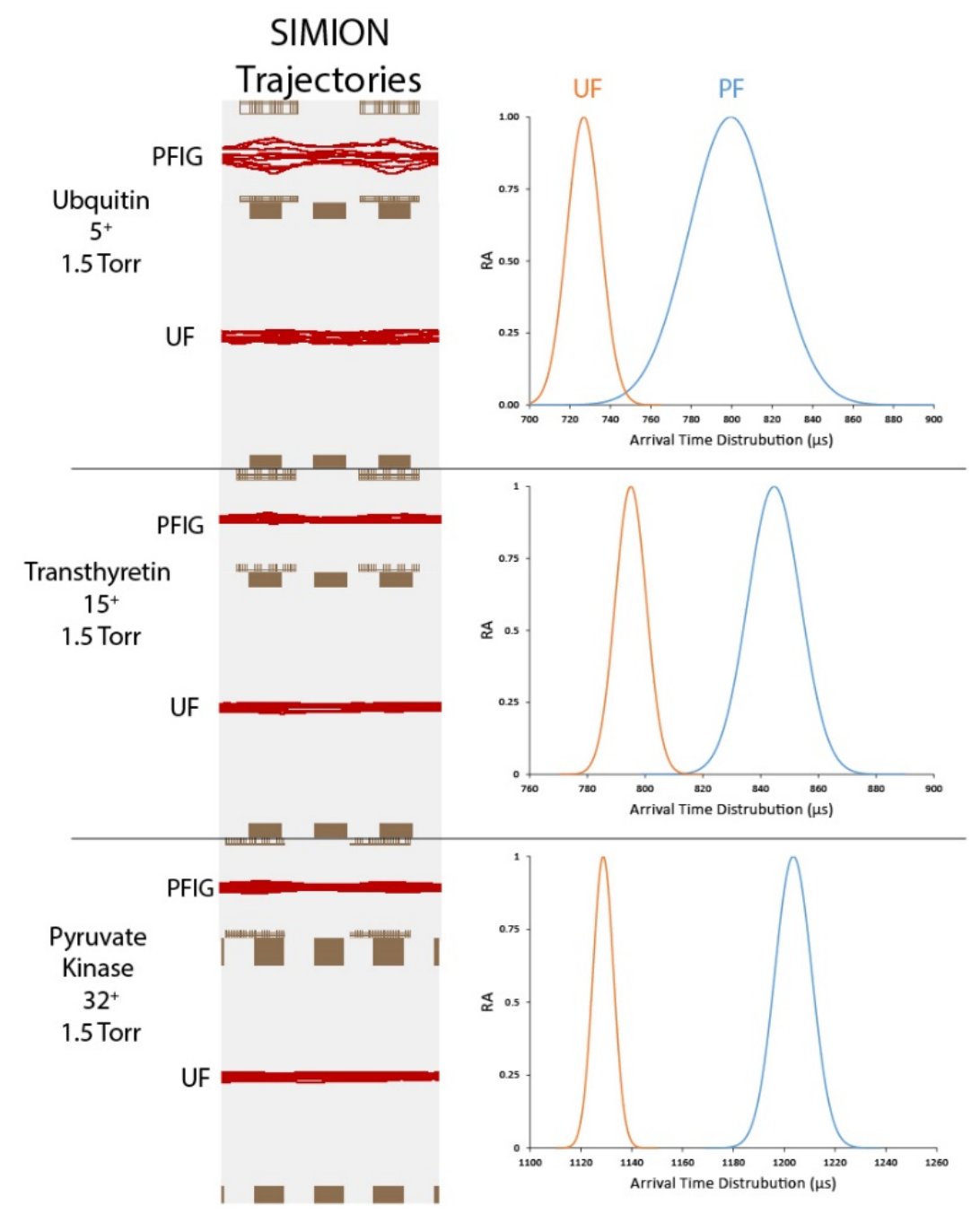

Figure S1: a factor determination via SIMION 8.1. 30 ions of different mass and CCS are simulated in a $10 \mathrm{~cm}$ uniform field (UF) and periodic focusing (DT). The average and standard deviation of the arrival time for the ions are fitted to a Gaussian peak. The ratio of the arrival times of UF vs PF gives rise to the calculated $\alpha$ value. The respective siimulations were performed at multiple pressures to determine $\alpha_{\mathrm{E} / \mathrm{N}}$. 
Table S1: Proteins and sources with abbreviations.

\begin{tabular}{|c|c|c|c|c|c|c|}
\hline Protein & Abbrev & $\mathbf{n}$ & $\begin{array}{c}\text { Molecular } \\
\text { Weight } \\
\text { (kDa) }\end{array}$ & Source & Manufacture & $\begin{array}{l}\text { Product } \\
\text { Number }\end{array}$ \\
\hline Ubiquitin & Ubq & 1 & 8.558 & $\begin{array}{l}\text { bovine } \\
\text { erythrocytes }\end{array}$ & Sigma & U6253 \\
\hline Lysozyme & Lyso & 1 & 14.30 & $\begin{array}{l}\text { Chicken Egg } \\
\text { White }\end{array}$ & Fisher & BP535 \\
\hline Myoglobin & Мyo & 1 & 17.56 & $\begin{array}{l}\text { equine skeletal } \\
\text { muscle }\end{array}$ & Sigma & M0630 \\
\hline$\beta$-Lactoglobulin A & BLG & 1,2 & $18.35,36.71$ & bovine milk & Sigma & L7880 \\
\hline Streptavidin & Strept & 4 & 51.88 & S. avidinii & ThermoFisher & 434301 \\
\hline Transthyretin & TTR & 4 & 55.75 & Recombinant & $\operatorname{Ref}^{1}$ & --- \\
\hline Hemoglobin & Hemo & 4 & 64.48 & Horse Heart & Sigma & $\mathrm{H} 4632$ \\
\hline $\begin{array}{l}\text { Bovine Serum } \\
\text { Albumin }\end{array}$ & BSA & 1 & 68.81 & Bovine & Sigma & 05470 \\
\hline Concanavalin A & ConA & 4 & 102.9 & $\begin{array}{l}\text { Canavalia } \\
\text { ensiformis }\end{array}$ & Sigma & C2010 \\
\hline $\begin{array}{c}\text { Alcohol } \\
\text { Dehydrogenase }\end{array}$ & $\mathrm{ADH}$ & 4 & 148.2 & S. cerevisiae & Sigma & A7011 \\
\hline Aldolase & Aldo & 4 & 157.1 & rabbit muscle & Sigma & A2714 \\
\hline Pyruvate Kinase & PK & 4 & 233.4 & rabbit muscle & Sigma & P9136 \\
\hline GroEL & GroEL & 14 & 810.1 & Recombinant & $\operatorname{Ref}^{2}$ & --- \\
\hline
\end{tabular}


Table S2: CCS and $\mathrm{K}_{\mathrm{o}}$ values calculated via PF-DT in helium (99.999\%) for all experimental charge states. * denotes partially unfolded conformation.

\begin{tabular}{|c|c|c|c|c|c|c|c|c|c|}
\hline Protein & $\begin{array}{l}\text { Mass } \\
(\mathrm{kDa})\end{array}$ & Pressure & $\mathbf{z}(+)$ & $\boldsymbol{\alpha}_{\mathbf{E} / \mathbf{N}}$ & $\begin{array}{c}\text { PF-DT } \\
\text { CCS } \\
\left(\mathbf{n m}^{2}\right) \\
\end{array}$ & $\begin{array}{c}\mathbf{u}(\mathbf{C C S}) \\
(\%) \\
\end{array}$ & $\begin{array}{c}K_{0} \\
\left(\mathrm{~cm}^{2} / \mathrm{V} \cdot \mathrm{s}\right) \\
\end{array}$ & $\mathbf{K}_{\mathbf{0}} / \mathbf{z}$ & $\begin{array}{c}\text { CCS Lit } \\
\left(\mathbf{n m}^{2}\right)\end{array}$ \\
\hline \multirow{3}{*}{ Ubq } & \multirow{3}{*}{8.558} & \multirow{3}{*}{1.725} & 4 & \multirow{3}{*}{0.87} & 10.8 & 3.04 & 1.73 & 4.32E-01 & 10.0 \\
\hline & & & 5 & & 11.4 & 3.17 & 2.06 & 4.11E-01 & 10.3 \\
\hline & & & $6^{*}$ & & 16.3 & 2.55 & 1.73 & $2.88 \mathrm{E}-01$ & 15.8 \\
\hline \multirow{3}{*}{ Lyso } & \multirow{3}{*}{14.30} & \multirow{3}{*}{1.725} & 6 & \multirow{3}{*}{0.87} & 14.2 & 1.94 & 1.97 & $3.28 \mathrm{E}-01$ & 13.6 \\
\hline & & & 7 & & 15.1 & 2.56 & 2.16 & $3.09 \mathrm{E}-01$ & 13.6 \\
\hline & & & $8 *$ & & 19.2 & 4.37 & 1.94 & $2.43 \mathrm{E}-01$ & 17.8 \\
\hline \multirow{2}{*}{ Мyo } & \multirow{2}{*}{17.56} & \multirow{2}{*}{1.725} & 7 & \multirow{2}{*}{0.87} & 17.7 & 2.57 & 1.83 & $2.61 \mathrm{E}-01$ & 17.2 \\
\hline & & & 8 & & 20.7 & 2.07 & 1.89 & $2.37 \mathrm{E}-01$ & 18.2 \\
\hline \multirow{2}{*}{ BLG } & \multirow{2}{*}{18.35} & \multirow{2}{*}{1.725} & 7 & \multirow{2}{*}{0.87} & 17.8 & 1.90 & 1.83 & $2.62 \mathrm{E}-01$ & 16.6 \\
\hline & & & 8 & & 18.2 & 2.72 & 2.06 & $2.57 \mathrm{E}-01$ & 16.9 \\
\hline 2 BLG & 36.71 & 1.725 & 12 & 0.87 & 29.5 & 1.20 & 1.83 & $3.06 \mathrm{E}-01$ & 29.0 \\
\hline \multirow{4}{*}{ Strept } & \multirow{4}{*}{51.88} & \multirow{4}{*}{2.085} & 13 & \multirow{4}{*}{0.89} & 34.9 & 1.03 & 1.78 & $1.37 \mathrm{E}-01$ & 33.4 \\
\hline & & & 14 & & 36.9 & 1.38 & 1.81 & $1.30 \mathrm{E}-01$ & 33.5 \\
\hline & & & 15 & & 37.5 & 0.82 & 1.91 & $1.27 \mathrm{E}-01$ & 33.7 \\
\hline & & & 16 & & 37.1 & 2.02 & 2.06 & $1.29 \mathrm{E}-01$ & - \\
\hline \multirow{3}{*}{ TTR } & \multirow{3}{*}{55.75} & \multirow{3}{*}{2.085} & 14 & \multirow{3}{*}{0.89} & 36.3 & 0.53 & 1.83 & $1.30 \mathrm{E}-01$ & 34.1 \\
\hline & & & 15 & & 36.2 & 0.50 & 1.96 & $1.31 \mathrm{E}-01$ & 34.0 \\
\hline & & & 16 & & 36.3 & 0.50 & 2.08 & $1.30 \mathrm{E}-01$ & 33.8 \\
\hline \multirow{2}{*}{ Hemo } & \multirow{2}{*}{64.48} & \multirow{2}{*}{2.085} & 16 & \multirow{2}{*}{0.89} & 40.3 & 1.04 & 1.91 & $1.19 \mathrm{E}-01$ & 43.2 \\
\hline & & & 17 & & 40.6 & 0.63 & 2.01 & $1.18 \mathrm{E}-01$ & 43.2 \\
\hline & & & 14 & & 45.7 & 1.88 & 1.49 & $1.06 \mathrm{E}-01$ & 40.9 \\
\hline BSA & 68.81 & 1.786 & 15 & 0.90 & 45.8 & 0.76 & 1.60 & $1.06 \mathrm{E}-01$ & 41.0 \\
\hline & & & 16 & & 45.9 & 1.25 & 1.70 & $1.06 \mathrm{E}-01$ & 40.6 \\
\hline & & & 18 & & 57.7 & 0.50 & 1.50 & $8.35 \mathrm{E}-02$ & - \\
\hline ConA & 1029 & 2085 & 19 & 090 & 57.9 & 0.50 & 1.58 & 8.32E-02 & - \\
\hline & & 2.000 & 20 & 0.90 & 57.8 & 0.41 & 1.67 & 8.33E-02 & 55.5 \\
\hline & & & 21 & & 57.9 & 0.39 & 1.75 & 8.31E-02 & 55.5 \\
\hline & & & 23 & & 73.6 & 0.52 & 1.51 & 6.29E-02 & 69.4 \\
\hline $\mathrm{ADH}$ & 1482 & 2085 & 24 & 000 & 73.0 & 0.54 & 1.57 & 6.29E-02 & 69.4 \\
\hline & 140.2 & 2.000 & 25 & 0.30 & 73.6 & 0.50 & 1.63 & $6.27 \mathrm{E}-02$ & 69.4 \\
\hline & & & 26 & & 73.7 & 0.50 & 1.69 & $6.26 \mathrm{E}-02$ & 68.3 \\
\hline
\end{tabular}


Table S2 (continued)

\begin{tabular}{|c|c|c|c|c|c|c|c|c|c|}
\hline \multirow{4}{*}{ Aldo } & \multirow{4}{*}{157.1} & \multirow{4}{*}{2.085} & 23 & \multirow{4}{*}{0.90} & 79.8 & 0.80 & 1.39 & $6.04 \mathrm{E}-02$ & 76.1 \\
\hline & & & 24 & & 80.1 & 0.41 & 1.44 & 6.02E-02 & 76.9 \\
\hline & & & 25 & & 80.1 & 0.54 & 1.50 & $6.01 \mathrm{E}-02$ & 77.2 \\
\hline & & & 26 & & 81.3 & 1.97 & 1.54 & 5.93E-02 & 77.3 \\
\hline \multirow{4}{*}{ PK } & \multirow{4}{*}{233.4} & \multirow{4}{*}{1.558} & 31 & \multirow{4}{*}{0.91} & 109.7 & 1.70 & 1.38 & $4.46 \mathrm{E}-02$ & 103. \\
\hline & & & 32 & & 109.9 & 0.50 & 1.42 & $4.45 \mathrm{E}-02$ & 103. \\
\hline & & & 33 & & 110.0 & 0.60 & 1.47 & $4.45 \mathrm{E}-02$ & 102. \\
\hline & & & 34 & & 110.1 & 0.85 & 1.52 & $4.46 \mathrm{E}-02$ & 102. \\
\hline \multirow{10}{*}{ GroEL } & \multirow{10}{*}{810.1} & \multirow{10}{*}{1.645} & 61 & \multirow{10}{*}{1.00} & 231.1 & 2.10 & 1.42 & 2.33E-02 & - \\
\hline & & & 62 & & 237.6 & 2.04 & 1.40 & $2.26 \mathrm{E}-02$ & - \\
\hline & & & 63 & & 239.8 & 2.01 & 1.41 & $2.24 \mathrm{E}-02$ & - \\
\hline & & & 64 & & 239.2 & 0.95 & 1.44 & $2.25 \mathrm{E}-02$ & - \\
\hline & & & 65 & & 240.4 & 0.96 & 1.45 & 2.24E-02 & - \\
\hline & & & 66 & & 240.8 & 1.49 & 1.47 & $2.23 \mathrm{E}-02$ & - \\
\hline & & & 67 & & 241.8 & 0.50 & 1.49 & 2.22E-02 & 209. \\
\hline & & & 68 & & 242.5 & 0.58 & 1.51 & 2.22E-02 & 209. \\
\hline & & & 69 & & 243.8 & 0.50 & 1.52 & $2.20 \mathrm{E}-02$ & 207. \\
\hline & & & 70 & & 246.7 & 1.16 & 1.53 & $2.18 \mathrm{E}-02$ & 207. \\
\hline
\end{tabular}



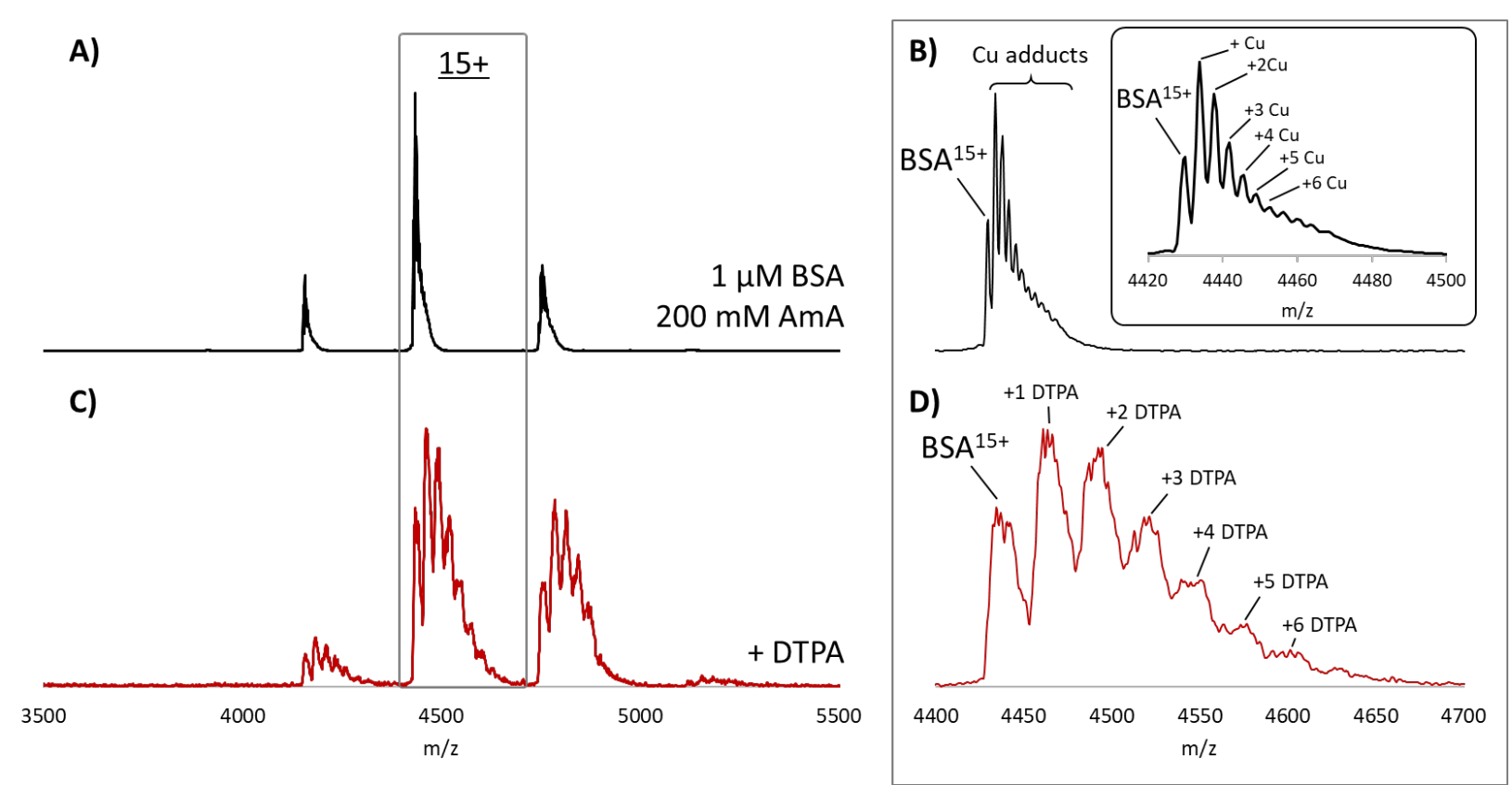

Figure S2. A) Resulting high resolution MS spectra of native BSA results in charge state distributions centered on $15^{+}$. B) Expansion of the $15^{+}$mass distributions reveal a host of adducts with an average molecular weight of $c a$. $60 \mathrm{Da}$, identified as possible $\mathrm{Cu}$ adducts (inset). C) Addition of a known $\mathrm{Cu}$ chelator (DTPA, diethylenetriamine pentaacetic acid), results in a slight shift towards lower average charge and a host of adducts. D) Expansion of the BSA ${ }^{15+}$ distribution reveals up to 6 DTPA adducts. 


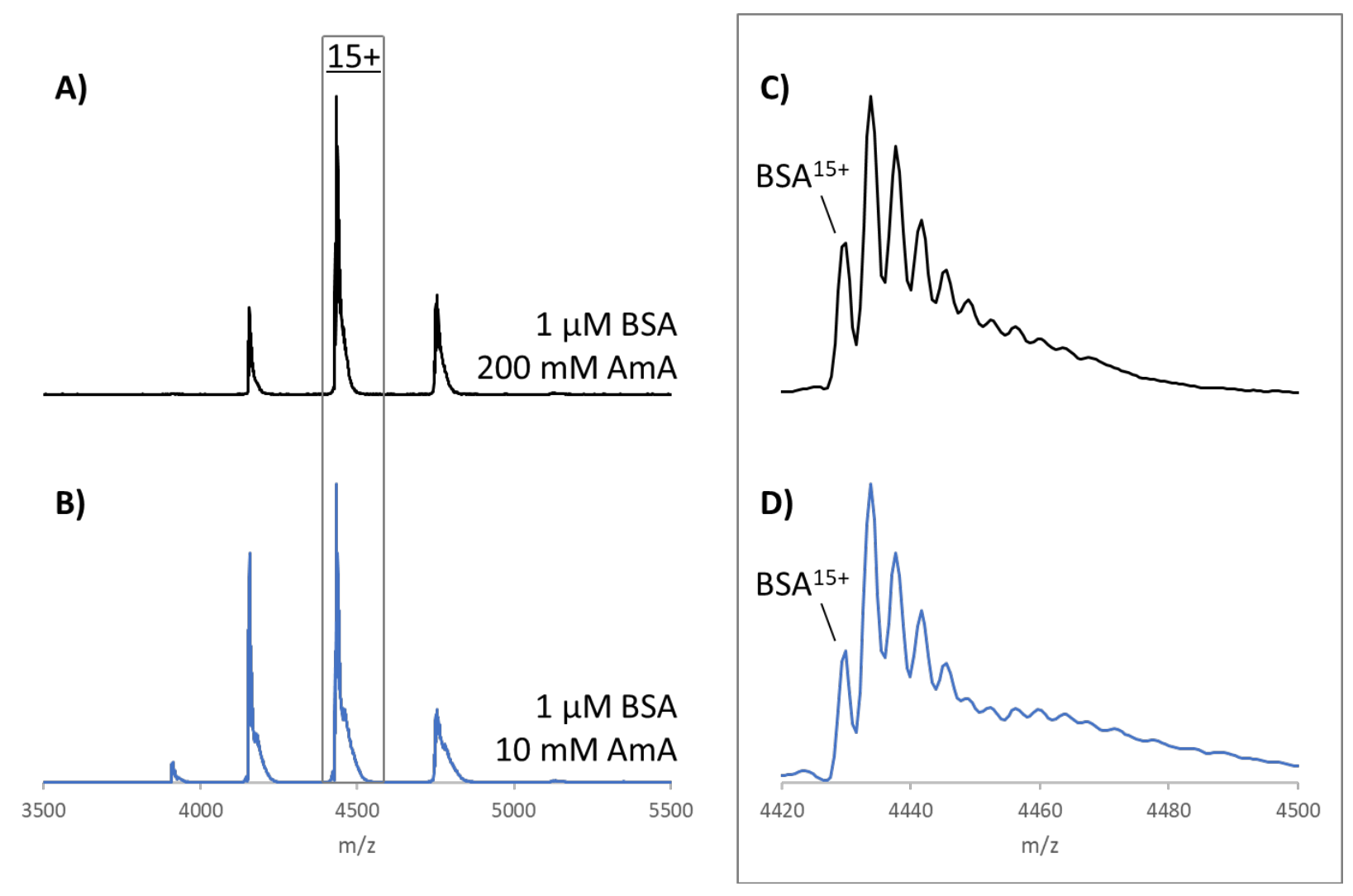

Figure S3. A) Resulting high resolution MS spectra of native BSA in $200 \mathrm{mM}$ ammonium acetate $(\mathrm{AmA})$ results in charge state distributions centered on $15^{+}$. B) Decreasing the AmA concentration to $10 \mathrm{mM}$ results in a slight shift towards higher average charge state. Expansion of the $\mathrm{BSA}^{15+}$ ion distributions from (C) $200 \mathrm{mM}$ and (D) $10 \mathrm{mM}$ AmA show qualitatively similar relative abundances of $\mathrm{Cu}$ adduction. 


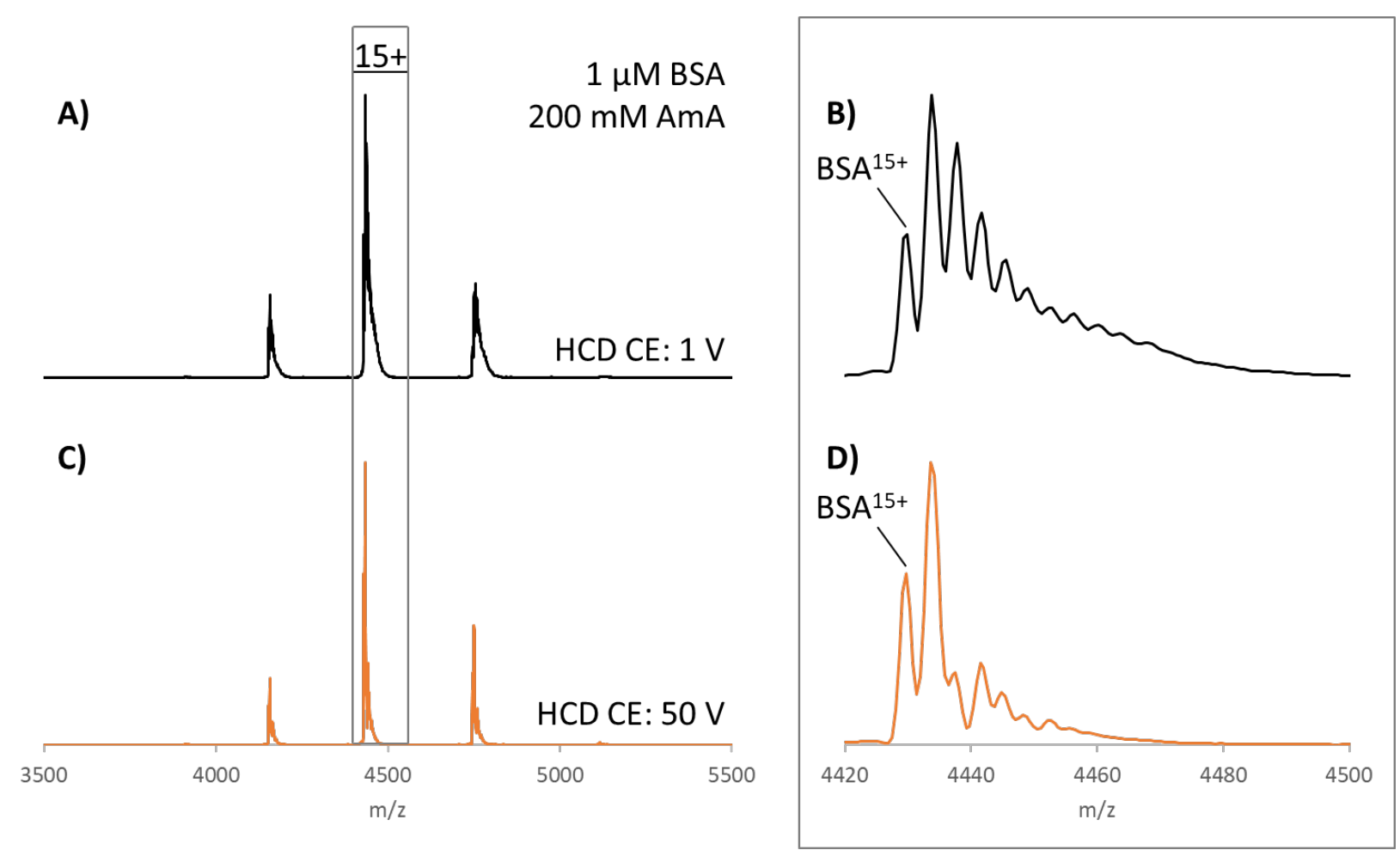

Figure S4. A) Resulting high resolution MS spectra of native BSA in $200 \mathrm{mM}$ ammonium acetate $(\mathrm{AmA})$ results in charge state distributions centered on $15+$. B) Expansion of the $\mathrm{BSA}^{15+}$ spectra reveals several $c a$. $60 \mathrm{Da}$ adducts. C) Increasing the HCD collision energy to $50 \mathrm{~V}$ does not significantly affect the observed charge state distribution. D) Expansion of the collisionally activated $\mathrm{BSA}^{15+}$ signal reveals fewer adducts. 

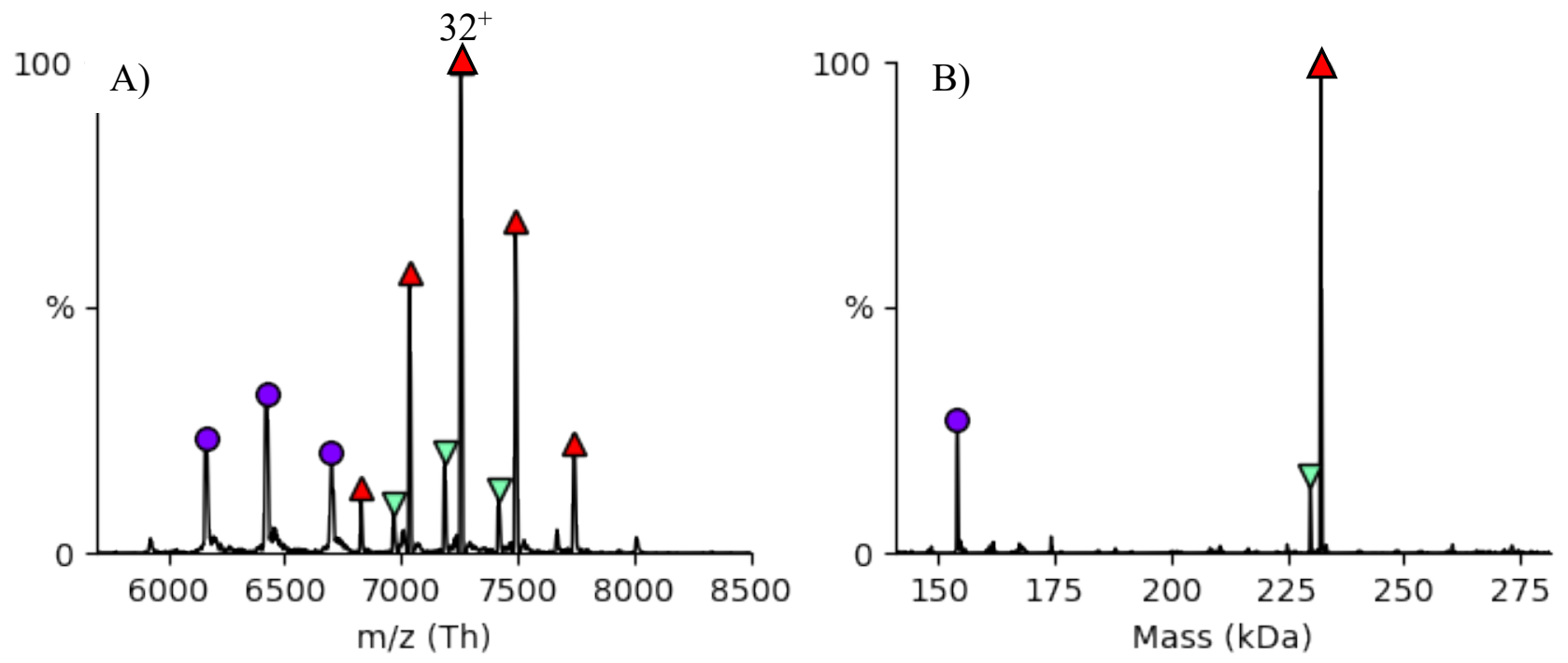

Figure S5. A) Resulting high resolution MS spectra of native pyruvate kinase in $200 \mathrm{mM}$ ammonium acetate (AmA) results in charge state distributions centered on 32+. B) Deconvoluted MS of PK with the resulting masses: 154.1, 229.9, 232.2 kDa showing dirty samples that can be resolved by higher MS resolution. 
Table S3: MS data for $1 \mu \mathrm{M}$ GroEL in $200 \mathrm{mM}$ ammonium acetate (AmA) obtained on different instruments.

\begin{tabular}{|c|c|c|c|c|}
\hline \multirow{2}{*}{ Charge State } & \multicolumn{4}{|c|}{ GroEL Observed Mass } \\
\cline { 2 - 5 } & UHMR* & UHMR REIS† & IM EMR‡ & EMR REIS† \\
\hline 75 & & 801993.0 & & \\
\hline 74 & & 802046.8 & & \\
\hline 73 & 800917.3 & 802001.4 & & 804825.4 \\
\hline 72 & 800904.2 & 802005.8 & & 804930.6 \\
\hline 71 & 800917.6 & 802060.7 & & 805072.8 \\
\hline 70 & 800926.7 & 802068.1 & 810649.7 & 805538.4 \\
\hline 69 & 800921.0 & 802133.3 & 809940.6 & 805577.6 \\
\hline 68 & 800933.2 & 802193.3 & 809914.7 & 805502.1 \\
\hline 67 & 800940.1 & 802272.1 & 810259.1 & 805729.3 \\
\hline 66 & 800950.9 & 802567.3 & 810454.3 & 805684.8 \\
\hline 65 & 800953.4 & 802595.3 & 810432.4 & 806036.5 \\
\hline 64 & 800955.5 & & 810046.7 & 806665.9 \\
\hline 63 & & & 809965.2 & 806290.8 \\
\hline 62 & & & 810059.1 & \\
\hline 61 & & & 810315.5 & \\
\hline 60 & & & 810570.6 & \\
\hline \%RSD & 0.0021 & 0.0259 & 0.0313 & 0.0665 \\
\hline
\end{tabular}

* UHMR MS data were collected under the default GroEL tune file via the commercial ionization source.

$\dagger$ Both the UHMR and the EMR were modified in a similar fashion as previously described to optimize tuning and sensitivity while having gentle tune conditions. ${ }^{3}$

+ IM-EMR data is collected via the optimized conditions with minimal heating to preserve native-like conformations.

\section{References:}

1. Shirzadeh, M.; Boone, C. D.; Laganowsky, A.; Russell, D. H., Analytical Chemistry 2019, 91 (3), 2345-2351.

2. Weaver, J.; Jiang, M.; Roth, A.; Puchalla, J.; Zhang, J.; Rye, H. S., Nature Communications 2017, 8 (1), 15934.

3. Poltash, M. L.; McCabe, J. W.; Patrick, J. W.; Laganowsky, A.; Russell, D. H., Journal of the American Society for Mass Spectrometry 2019, 30 (1), 192-198. 